East African Medical Journal Vol. 80 No. 7 July 2003

CRYPTOSPORIDIUM OOCYSTS IN GHANAIAN AIDS PATIENTS WITH DIARRHOEA

A. Adjei, PhD, Asociate Professor, Department of Pathology, M. Lartey, MBChB, FWACP, Lecturer, Department of Medicine, T.K. Adiku, PhD, Consultant, Department of Microbiology, University of Ghana Medical School, Korle-Bu, Accra, O. Rodrigues, MBChB, FWACP, Consultant, Department of Child Health, Korle-Bu Teaching Hospital, Korle-Bu, Accra, L. Renner, MBChB, FWACP, Consultant, Department of Child Health, Korle-Bu Teaching Hospital, Korle-Bu, Accra, E. Sifah, MBChB, FWACP, Specialist, Department of Child Health, Korle-Bu Teaching Hospital, Korle-Bu, Accra, Ghana, J.D. Mensah, MBChB, FWACP, Principal Medical Officer, KorleBu Polyclinic, Korle-Bu, Accra, Ghana, B. D. Akanmori, PhD, Asociate Professor, J. Otchere, Principal Medical Technician, B.K. Bentum, BSc, Research Assistant and K.M. Bosompem, PhD, Senior Research Fellow, Noguchi Memorial Institute for Medical Research, Legon, Accra, Ghana

Request for reprints to: Dr. K.M. Bosompem, Noguchi Memorial Institute for Medical Research, P.O. Box LG581, Legon, Accra, Ghana

\title{
CRYPTOSPORIDIUM OOCYSTS IN GHANAIAN AIDS PATIENTS WITH DIARRHOEA
}

\author{
A. ADJEI, M. LARTEY, T.K. ADIKU, O. RODRIGUES, L. RENNER, E. SIFAH, J.D. MENSAH, \\ B. AKANMORI, J. OTCHERE, B.K. BENTUM and K.M. BOSOMPEM
}

\begin{abstract}
Background: AlthoughCryptosporidium spp. infections in acquired immunodeficiency syndrome patients (AIDS) with chronic diarrhoea have been reported in several African countries, there is no information regarding cryptosporidial diarrhoea in Ghanaian AIDS patients.

Objective: To investigate the occurrence of $C$. parvum and other gastrointestinal parasitic agents in Ghanaian AIDS patients with chronic diarrhoea.

Design: Prospective study of HIV/AIDS patients with diarrhoea over a nine month period. Setting: Korle-Bu Teaching Hospital and Korle-Bu Polyclinic Accra, Ghana.

Results: Analysis of stool specimens from clinically diagnosed HIV/AIDS (n=2I; mean CD4 count was 288 cells per microliter, $95 \%$ confidence interval of 237 to 340 cells per microliter) and HIV-seronegative (n=27) patients revealed $C$. parvum in six $(28.6 \%)$ of HIV/AIDS and $10(37.0 \%)$ of the HIV-seronegative patients, respectively. Three other HIV/AIDS cases had other infections involving Strongyloides stercoralis $4.8 \%$ (1/21) and Salmonella spp. 9.5\% (2/21). There was no concomitant association between $C$. parvum and any other parasites found. Also, no enterobacteria was found in the HIV-seronegative patients.

Conclusion: This study demonstrates the prevalence of Cryptosporidium sp. in both HIV/ AIDS and HIV-seronegative individuals in Ghana. However, there was no statistical association between cryptosporidiosis and HIV/AIDS $(p>0.05)$.
\end{abstract}

\section{INTRODUCTION}

Enteric cryptosporidiosis, caused by the coccidian parasite Cryptosporidium sp., has become of increasing interest since the first case of human cryptosporidiosis was reported in 1976(1). Much of this interest is due to its opportunistic behaviour in immunocompromised patients, particularly in those with acquired immune deficiency syndrome (AIDS) $(2,3)$. AIDS predisposes individuals to several parasitic diseases, and among the intestinal parasites, the intracellular coccidia, Isospora belli and Cryptosporidium parvum, are important(4). As a result of increased cryptosporidial related diarrhoea in HIV/AIDS patients, the disease was recently listed as one of the AIDS-defining opportunistic infections(5).

Cryptosporidiosis develops in an estimated 10-15\% of patients with AIDS in the United States of America and Europe $(3,6,7)$ and $30-50 \%$ of patients with AIDS in the developing countries $(8,9)$. Previous studies in Ethiopia, Zambia, Democratic Republic of Congo and other African countries have shown high prevalence rates of $C$. parvum infections in AIDS patients(10-12). The role of cryptosporidiosis in HIV/AIDS related diarrhoea in Ghana is virtually unknown. With a continuous rise in the number of HIV/AIDS infected persons, and with a prevalence rate currently estimated at $4 \%$ (National AIDS/STD Control Programme, Ministry of Health, Accra, Ghana), It is likely that Cryptosporidium sp. related diarrhoea may be prevalent in Ghana. This is even more likely because various animals that are potential sources of transmission (dogs, cats, cattle and pigs) and in some cases rats and mice share human habitations. Additionally, the common sources of drinking water including tap water, may be contaminated because of inadequacy of standard water treatment methods to remove the parasite. The aim of this study was to determine the prevalence of Cryptosporidium and other gastrointestinal parasitic agents in Ghanaian AIDS patients with chronic diarrhoea.

\section{MATERIALS AND METHODS}

Study area and subjects. This study was conducted at the Fevers Unit and the Polyclinic Unit of the Korle-Bu Teaching Hospital, Accra, Ghana, between February and September, 2001. Korle-Bu Teaching Hospital is a leading tertiary hospital which serves the city of Accra, surrounding urban population and the southern part of Ghana. Accra, the capital city of Ghana is a rapidly expanding city with a population of about 2 million. Twenty one HIV/AIDS patients (aged 8 months 54 years 11 
males and 10 females) with chronic diarrhoea on admission at the Fevers Unit, and twenty seven HIV-seronegative individuals (control; aged 6-60 years 16 males and 11 females) who reported at the Polyclinic Unit with chronic diarrhoea were recruited in the study. The protocol for this study was approved by the Ethical and Protocol Review Committee of the University of Ghana Medical School, and written and informed consent was obtained from the patients. Following informed consent, a detailed demographic questionnaire including history and clinical status was completed with the help of a study nurse. The guidelines for the classification of HIV/AIDS in adults from the Centers for Disease Control (CDC), USA (13) were applied to both groups of patients. HIV testing was done by the particle agglutination test (Serodia HIV-1 and HIV-2: Fujirebio inc., Tokyo, Japan ) and confirmed by Western Blot analysis (New Lav Blot I and II) or the synthetic peptide-based immunoassay (PeptiLav I and II), both obtained from Sanofi Diagnostic Pasteur, Marnes-laCoquette, France.

Definition of diarrhoea episodes: The World Health Organisation (WHO) criteria was used to determine diarrhoeal episodes (14). A total of three or more unformed stools in one 24hour period was determined to be a diarrhoeal day.

Specimen collection and processing: Diarrhoeal samples from each patient was collected directly into sterile disposable containers and a portion of each sample subsequently preserved in a tube containing $10 \%$ formalin for parasitological examination. Fresh stools were transported to the laboratory for parasitologic and microbiologic evaluation within 24 hours. Preserved stool specimens were concentrated using formalin-ethyl acetate at $800 \mathrm{xg}$ in a faecal parasite concentrator and two slides were made from the resulting pellet. One slide was stained with Lugol's iodine and examined microscopically at 400x magnification for the presence of endemic parasites: Giardia lamblia cysts, Trichuris trichiura, Ascaris lumbricoides, Strongyloides stercoralis, Ancylostoma spp., Schistosoma mansoni and Entamoeba spp. The second slide prepared with faecal pellet was used to detect $C$. parvum oocysts by modified Ziehl Neelsen technique. Briefly, slides were stained for $20 \mathrm{~min}$ with carbol fucshin and de-stained for 30-60 seconds with a 10\% solution of sulphuric acid. After washing, slides were counterstained with methylene blue for $1 \mathrm{~min}$. Red-stained $C$. parvum oocysts were observed microscopically using a 40x objective. Fresh wet stool preparations stained with Lugol's iodine were also examined for parasites. Unstained, fresh stool specimen were plated onto various bacteriological agar media to isolate bacteria: MacConkey
(Shigella species, Salmonella species) xylose-lysinedeoxycholate (Yersiniaenterocolitica). thiosulphate-citrate-bilesucrose, trypticase soy with 5\% defibrinated sheep blood and ampicillin(Campylobacter jejuni). Culturing for enterotoxigenic Escherichia coli and demonstration of rotavirus could not be performed.

Statistical analysis: Statistical analysis were performed by Fisher's exact or Wilcoxon rank test. Results were considered significant when the P-value was less than 0.05 .

\section{RESULTS}

All the $21 \mathrm{HIV} / \mathrm{AIDS}$ (with a single infection from HIV-1) patients fell within the CDC clinical staging AIC3 categories, representing asymptomatic AIDS to severe AIDS conditions. The patients had mean CD4 counts of 288 cells per microliter ( $95 \%$ confidence interval of 237 340 cells per microliter).

All patients reported watery stools lasting from 3-90 days (diarrhoea episodes, 3-10 stools/day). In addition to diarrhoea, the most common symptoms in both groups of patients (HIV/AIDS and HIV-negative patients) included abdominal pain, nausea, vomiting and fever. Presence of blood in stools was, less frequently reported (Table 1).

As shown in Table 1, significantly more HIV-negative patients $11(40.7 \%)$ had abdominal pains compared to HIV/AIDS patients $5(23.8 \%)(\mathrm{p}<0.05)$. Nevertheless, none of the HIV/AIDS patients with abdominal pain and two out of 11 HIV-negative patients had Cryptosporidium oocysts in their stools. Of the 21 HIV/AIDS and 27 HIVnegative patients, six $(28.6 \%)$ and $10(37.0 \%)$, respectively had $C$. parvum only (Table 2 ).

There was no concomitant association between $C$ parvum and any other intestinal parasites found in the stools of both categories of patients. Of the remaining HIV/AIDS cases, one patient had S. stercoralis and two had Salmonella spp, however, no enterobacteria was found in the HIV-negative patients (Table 2). Major risk factors such as drinking of untreated/ unboiled water and close association with domestic animals (chickens, pigs, cattle, sheep, goats, dogs, cats) had no significant effect on Cryptosporidium infections ( $\mathrm{p}>0.05)$.

Table 1

Symptoms associated with chronic diarrhoea in HIV/AIDS and HIV-seronegative patients

\begin{tabular}{lccc}
\hline Characteristic & \multicolumn{2}{c}{ HIV/AIDS $(\mathrm{n}=21)$} & HIV-seronegative (n=27) \\
\cline { 2 - 4 } Symptom & Number $(\%)$ & $\begin{array}{c}\text { Cryptosporidium spp } \\
\text { positive }\end{array}$ & $\begin{array}{c}\text { Number (\%) } \\
\text { Cryptosporidium spp } \\
\text { positive }\end{array}$ \\
\hline Abdominal pain & $5(23.8)$ & 0 & $11(40.7)$ \\
Nausea & $9(42.8)$ & 2 & $12(44.4)$ \\
Vomiting & $7(33.3)$ & 3 & $9(33.3)$ \\
Fever & $8(38.1)$ & 2 & $13(48.1)$ \\
Blood in stool & $1(4.8)$ & 0 & $3(11.1)$
\end{tabular}

\footnotetext{
* Stool samples were loose and take the shape of the container
} 
Table 2

Pathogens identified in HIV/AIDS and HIV-seronegative patients with chronic diarrhoea

\begin{tabular}{lcc}
\hline Pathogen & $\begin{array}{c}\text { HIV/AIDS } \mathrm{n}=21 \\
(\%)\end{array}$ & $\begin{array}{c}\text { HIV-seronegative } \mathrm{n}=27 \\
(\%)\end{array}$ \\
\hline Cryptosporidium spp. & $6(28.6)$ & $10(37.0)$ \\
Strongyloides stercoralis & $1(4.8)$ & 0 \\
Salmonella spp. & $2(9.5)$ & 0 \\
\hline
\end{tabular}

\section{DISCUSSION}

Cryptosporidiosis is a highly infectious illness with multiple modes of transmission through water, person-toperson and zoonosis(16). It has a serologic prevalence as high as $30-60 \%$ in industrialised countries $(17,18)$ and $95 \%$ in some tropical and developing countries (16). Cryptosporidia are etiologic agents in 10-35\% of' cases of AIDS-associated diarrhoea, and cryptosporidiosis results in a significant morbidity and mortality in patients with AIDS world-wide(5,7). However, there is little or no information available on the prevalence, significance, and prognosis of Cryptosporidium infection in Ghana, particularly in HIV/AIDS patients.

In this study, Cryptosporidium parvum oocysts were identified by the modified Ziehl Neelsen method whose sensitivity and specificity are reported to be comparable to that of the immunofluorescence assay(19). C. parvum was isolated as the dominant pathogen from both HIV/AIDS and HIV seronegative groups of patients with diarrhoea than any of the other enteric pathogens investigated ( $<<0.05$ ). This finding suggested that Cryptosporidium may be an unrecognized pathogen of diarrhoea and other gastrointestinal disorders in the Ghanaian population. However, there is the need to determine the importance of other agents of diarrhoea, such as enteropathogenic $E$. $c o l i$, viral and microsporidia infections which were not investigated in this study.

The results of this study are in agreement with that of Lucas (1990) who noted that the more 'common' intestinal parasites, G. lamblia, T. trichiura, A lumbricoides and $S$. stercoralis are not opportunistic in HIV/AIDS patients (20), Even though Cryptosporidia and other diarrhoea-causing pathogens produce similar symptoms, Tzipori (1988) reported that abdominal pains is associated with cryptosporidial diarrhoea(21). Nevertheless, the absence of abdominal pains in most Cryptosporidium infected diarrhoeal patients in this study may therefore suggest other causes of diarrhoea. Cryptosporidial diarrhoea is self-limiting in immunocompetent hosts and may be found in active disease. Furthermore, the lack of clear association with possible risk factors of acquiring cryptosporidiosis (such as consumption of contaminated drinking water and contact with domestic animals) calls for more work to identify the major routes of infection in the study area.
In summary, the data presented herein demonstrates the occurrence of Cryptosporidium among HIV/AIDS and HIV-seronegative diarrhoeal patients in Ghana. The disease was however not significantly associated with HIV/AIDS, and the high prevalence of infection in the HIV-seronegative population suggested that the transmission of Cryptosporidium in the study communities is high. In view of the increasing prevalence of HIV/AIDS in Ghana, findings from future studies will be useful in developing preventive measures for reducing infection among immunodeficient groups.

\section{ACKNOWLEDGEMENTS}

We appreciate the support of Dr. J. Wastling, Institute of Biomedical and life Science, University of Glasgow, U.K, and other contribution: Prof. H. V Smith, Director, Scottish Parasite Diognostic Laboratory, Stobhil Hospital, Glasgow, Miss Alison Shutt formely of the same laboratory as well as Dr. Annette Macleod and Mrs Marianne Mallon both of Wellcome Unit of Molecular Parasitology, University of Glasgow. This project was sponsored by the College of Health Sciences, University of Ghana and the British Council under the Higher Education Link Programme.

\section{REFERENCES}

1. Nime, F.A.,. Burek, J.D., Page, D.L., Holscher, M.A. and Yardley, J.H. Acute enterocolitis in a human being infected with the protozoan Cryptosporidium. Gastroenterol. 1976; 70: $592-598$

2. McGowan, L., Hawkins, A.S. and Walker, I.V.D. The natural history of cryptosporidial diarrhoea in HIV infected patients. AIDS .1993; 7: 349-354.

3. Peterson, C, Cryptosporidosis in patients infected with human immunodeficiency virus. Clin. Infect. Dis. 1992;15:903-909.

4. Fleming, A.F. Opportunistic infections in AIDS in developed and developing countries.Trans. Roy. Trop. Med. Hyg. 1990; 84 (Suppl 1): 1-5.

5. J. Kuby The Immune System in AIDS, In. imunology. ed., 1997; pp523-554, . WH Freeman and Company, New York, USA.

6. Laughon, B.E., Druckman, D.A. and Vernon, A.A. Prevalence of enteric pathogens in homosexual men and without acquired immunodeficiency syndrome. Gastroenterol. 1988; 94: 984993.

7. Colford, J.M.,Tager, I.B., Hirozawa, A.M., Lemp, G.F., Aragon,T. and Petersen, C. Cryptopsoridiosis among patients infected with human immunodeficiency virus. Am. J. Epidemiol. 1996; 144: 807-816.

8. Sanchez-Mejorand, G. and Ponce-de-Leon, S, Clinical patterns of diarrhoea: etiology and prognosis. Rev. Invest. Clin. 1994; 46: 187-196.

9. Wuhib, T., Siva, T.M.J. and Newmann, R.D. Cryptosporidial and microsporidial infections in human immunodeficiency virus-infected patients in northeastern Brazil. J. Infect. Dis. 1994; 170: 494-497.

10. Fisseha, B., Petros, B. and Woldemichael, T. Cryptosporidium and other parasites in Ethiopian AIDS patients with chronic diarrhoea. E. Afr. Med. J. 1998; 75: 100-101.

11. Kelly. P., Sri Baboo. K., Ndubani, P., et al. Cryptosporidiosis in Adults in Lusaka, Zambia, and its relationship to oocysts contamination of drinking water. J. Infect. Dis. 1997; 176: 1120-1123. 
12. Colebunders, R. Persistent diarrhoea in Zairean AIDS patients: an endoscopic and histologic study. Gut 1990; 29: 1687 1691.

13. Levy, J.A. Revised classification systern for HIV infection and expanded AIDS surveillance case definition for adolescents and adults. In HIV and pathogenesis of AIDS. American Society of Microbiology pp 241-246, 1994, (ASM) Press, Washington. DC.

14. World Health Organization. Persistent diarrhoea in children in developing Countries: Memorandum from a WHO meeting. Bull WHO. 1988; 66: 708-717.

15. Dupont, H.L., Chappell, C.L. Sterling, C.R., Okhuysen. P.C. and Rose, J.B. The infectivity of Cryptosporidium parvum in healthy volunteers. N. Engl. J. Med 1995; 332:855-859.

16. Newman, R.D., Zu, S.X., Wuhib.T., Guerrant, R.L. and Sears, C.L. Hosehold epidemiology of Cryptosporidium parvum infection in an urban community in northeast Brazil. Ann. Intern. Med. 1994; 120: 500-505.

17. Goodgame, R.W. Understanding intestinal spore-forming protozoa: cryptosporidia, microsporidia, isospora and cyclospora. Ann. Intern. Med. 1996; 124: 1429-1441.

18. KuhIs, T.L., Mosier, D.A., Crawford, D.L. and Griffis, J. Seroprevalence of cryptosporidial antibodies during infancy, childhood, and adolescence. Clin. Infect. Dis. 1994; 18: 731735 .

19. Macpherson, D.W. and McQueen, R. Cryptosporidiosis: multiattribute evaluation of six diagnostic methods. J. Clin. Microbiol. 1993; 31: 198-202.

20. Lucas, S.B. Missing infection in AIDS. Trans. Roy. Soc. Trop. Med. Hyg. 1990; 84(Suppl): 34-38.

21. Tzipori, S. Cryptosporidiosis in perspective. Adv. Parasitol. 1988; 27:64-129. 